\title{
Nghiên cứu vai trò trung gian của sự cam kết trong mối quan hệ giữa nguồn lực tâm lý và kết quả làm việc của nhân viên tại khu vực Thành phố Hồ Chí Minh
}

\section{The mediating role of organizational commitment in the relationship between psychological capital and employee performance}

\author{
Lâm Hoàng Phương ${ }^{1 *}$, Nguyễn Văn Thụy ${ }^{2}$, Hồ Thị Kim Cương ${ }^{3}$, Lê Hoàng Quân ${ }^{4}$ \\ ${ }^{1}$ Trường Đại học Văn Lang, Việt Nam \\ ${ }^{2}$ Trường Đại học Ngân Hàng Thành phố Hồ Chí Minh, Việt Nam \\ ${ }^{3}$ Công ty gốm sứ Minh Long, Việt Nam \\ ${ }^{4}$ Công ty TM-DV-ĐT Lâm Gia, Việt Nam \\ *Tác giả liên hệ, Email: hoangphuong.hrc@gmail.com
}

THÔNG TIN

DOI: $10.46223 / \mathrm{HCMCOUJS}$. econ.vi.16.1.554.2021

Ngày nhận: 23/06/2020

Ngày nhận lại: 07/07/2020

Duyệt đăng: 04/09/2020

Tù khóa:

nguồn lực tâm lý, sự cam kết, kết quả công việc

\section{TÓM TẮT}

Kể từ khi lý thuyết về quản trị lần đầu tiên được giới thiệu bởi Taylor vào năm 1911 đến nay, các nhà khoa học cũng như các nhà quản trị đã thực hiện nhiều nghiên cứu khác nhằm giúp nâng cao kết quả làm việc của nhân viên. Các yếu tố ảnh hưởng đến kết quả làm việc của nhân viên ban đầu được xác định là các yếu tố bên ngoài liên quan đến các phần thưởng, môi trường làm việc, kỹ thuật... Tuy nhiên, trường phái tâm lý xã hội trong quan trị đã xuất hiện và đề cao vai trò của sự thỏa mãn các nhu cầu cơ bản của người lao động cũng như các yếu tố về tâm lý. Nghiên cứu này được thực hiện nhằm kiểm định mối quan hệ giữa nguồn lực tâm lý, cam kết tình cảm và kết quả công việc của nhân viên văn phòng tại khu vực Thành phố Hồ Chí Minh. Với mẫu khảo sát là 304, sau khi phân tích EFA, CFA, SEM thu được kết quả như sau: có tác động dương giữa nguồn lực tâm lý và cam kết tình cảm, giữa nguồn lực tâm lý và kết quả công việc. Song nghiên cứu cũng phát hiện rằng nếu nguồn lực tâm lý tác động đồng thời đến cam kết tình cảm và kết quả công việc thì cam kết tình cảm lại không có tác động đến kết quả làm việc.

\section{ABSTRACT}

Since the first theory of management was introduced by Taylor in 1911, many researchers as well as managers have conducted several types of researches to identify the factors having impact on employees' performance. At first, antecedent variables were identified such as rewards, working climate, technology... However, the emergence of the Social Psychological approach had indicated the essential role of satisfaction of employees' needs as well as psychological factors. This study was conducted to examine the relationships among Psychological Capital (PsyCap),
Keywords:

psychological capital, organizational commitment, job performance 
Affective Commitment and Employee's Performance. Sample with 304 respondents was collected. After conducting EFA, CFA and SEM, the result showed that there is a positive relationship between PsyCap and Affective Commitment, PsyCap and Job Performance. The study also indicated that Affective Commitment does not play a mediation role in the relationship between PsyCap and Job Performance.

\section{Giới thiệu}

Quá trình hội nhập khiến các doanh nghiệp cạnh tranh với nhau khốc liệt để thỏa mãn nhu cầu ngày càng cao của thị trường. Các doanh nghiệp trong nước ta không chỉ cạnh tranh với nhau mà còn phải cạnh tranh với các doanh nghiệp nước ngoài. Trước những thách thức vô cùng to lớn của môi trường kinh doanh hiện tại, mỗi doanh nghiệp cố gắng tạo ra cho mình một lợi thế cạnh tranh trên thị trường. Do đó, mọi nguồn lực của tổ chức đều có thể trở thành lợi thế riêng, một số doanh nghiệp sử dụng nguồn lực về tài chính, một số khác tập trung phát triển nguồn lực tri thức (Etebarian, Tavakoli, \& Abzari, 2012). Tuy nhiên, trong những thập niên gần đây nguồn nhân lực nhân lực lại trở thành tâm điểm cạnh tranh giữa các doanh nghiệp. Luthans, Luthans, và Luthans (2004) đã phát triển một khái niệm mới liên quan đến nguồn nhân lực - nguồn lực tâm lý - mà ông cho rằng đây chính là một bước tiến xa hơn trong việc xây dựng và đánh giá năng lực cạnh tranh của các doanh nghiệp hiện nay.

Các nghiên cứu trước đây về kết quả làm việc của nhân viên cho thấy có rất nhiều biến tác động bao gồm: phong cách lãnh đạo (Pradeep \& Prabhu, 2011), môi trường làm việc (Suliman \& Al Harethi, 2013), sự tự chủ trong công việc, tiền lương, bản chất công việc (Khan, Nawaz, Aleem, \& Hamed, 2012). Tuy nhiên, các biến tác động này thuộc yếu tố ngoại sinh - chịu sự tác động bên ngoài. Nghiên cứu của Peterson, Luthans, Avolio, Walumbwa, và Zhang (2011) đã cho thấy yếu tố nội sinh cũng ảnh hưởng đến kết quả làm việc của nhà viên mà cụ thể là yếu tố nguồn lực tâm lý. Nguồn lực tâm lý của nhân viên được hiểu là trạng thái tâm lý tích cực bao hàm 4 thành phần chính: Sự tự tin, sự lạc quan, niềm hi vọng và năng lực phục hồi (Luthans, Avolio, Avey, \& Norman, 2007). Không những có tác động trực tiếp đến kết quả làm việc của nhân viên, nguồn lực tâm lý còn gia tăng các các thái độ tích cực của nhân viên như: sự hài lòng trong công việc, sự cam kết với tổ chức, sự thoải mái về tâm lý; và các hành vi tích cực như hành vi công dân trong tổ chức. Đồng thời, nguồn lực tâm lý còn đóng vai trò quan trọng trong việc làm giảm các trạng thái tâm lý tiêu cực của nhân viên như: sự căng thẳng trong công việc, ý định nghỉ việc.

Vai trò của nguồn lực tâm lý trong mối quan hệ với kết quả công việc cũng như các biến thuộc thái độ và hành vi của nhân viên đã được chứng minh trong nhiều nghiên cứu trước đây. Mặc khác, sự cam kết với tổ chức giúp định hình những hành vi tích cực của nhân viên và giúp gia tăng kết quả làm việc (Hafiz, 2017). Tuy nhiên, sự tác động đồng thời giữa nguồn lực tâm lý và sự cam kết với tổ chức đến kết quả làm việc của nhân viên vẫn chưa được kiểm định tại Việt Nam. Do đó, nghiên cứu được thực hiện nhằm kiểm định vai trò trung gian của Sự cam kết với tổ chức trong mối quan hệ giữa nguồn lực tâm lý và kết quả làm việc của nhân viên tại khu vực thành phố Hồ Chí Minh. Kết quả nghiên cứu giúp bổ sung vào cơ sở lý thuyết về nguồn lực tâm lý và kết quả làm việc của nhân viên thông qua việc kiểm định vai trò của sự cam kết với tổ chức trong bối cảnh nên kinh tế đang phát triển mà cụ thể là Việt Nam.

\section{Cơ sở lý thuyết}

\subsection{Kết quả làm việc của nhân viên}

Kết quả làm việc của nhân viên là một trong những chỉ số đáng chú ý nhất trong việc đánh 
giá kết quả của tổ chức (Wall et al., 2004). Thuật ngữ này đề cập đến cảm nhận của cá nhân khi đã hoàn thành xong các yêu cầu công việc của họ trong tuần qua (Luthans et al., 2007). Munchinsky (2003) đề xuất cũng khái niệm: Kết quả công việc là một tập hợp các hành vi của nhân viên, có thể được đo lường, giám sát và đánh giá trong hệ thống thành tích ở cấp độ cá nhân.

Theo Porter và Lawler (1968), có ba hướng tiếp cận hay phương thức đo lường kết quả công việc của nhân viên. Một, sử dụng thước đo của mức giá đầu ra, số lượng bán hàng trong một thời gian nhất định, việc sản xuất của một nhóm các nhân viên báo cáo cho người quản lý,... Loại thứ hai liên quan đến xếp hạng của các cá nhân bởi một người khác. Và cuối cùng là tự đánh giá và tự nhận định. Nghiên cứu đã cho thấy rằng, việc áp dụng các kỹ thuật tự đánh giá và tự nhận định là hữu ích trong việc khuyến khích người lao động phát huy vai trò tích cực trong việc thiết lập mục tiêu của riêng mình.

\subsection{Nguồn lục tâm lý}

Đề cập đến vấn đề xa hơn của nguồn lực con người, không chỉ chú ý vào các yếu tố như trình độ, kinh nghiệm, kiến thức tiềm ẩn... mà còn tập trung hơn vào khía cạnh tâm lý tích cực được gọi là "nguồn lực tâm lý" (Luthans et al., 2007). Cụ thể, không chỉ quan tâm "bạn biết ai", "bạn biết gì" như nguồn vốn con người, nguồn lực tâm lý thể hiện "bạn là ai”, "người mà bạn sẽ trở thành và biến bản thân trở nên tốt nhất” (Luthans et al., 2007).

Nguồn lực tâm lý được xây dựng dựa trên những học thuyết và nghiên cứu trước, nổi bật là hành vi tích cực của tổ chức (Positive Organizational Behaviors - POB). Luthans và cộng sự (2007) cho rằng Nguồn lực tâm lý bao gồm bốn trạng thái tích cực:

(1) Sự tụ tin để gánh vác công việc và nỗ lực cần thiết để hoàn thành những nhiệm vụ mang tính thách thức;

(2) Sụ lạc quan tạo biểu hiện tích cực về những thành công trong cả hiện tại lẫn tương lai;

(3) Sự hy vọng kiên trì hướng tới mục tiêu và khi cần thiết có thể chuyển hướng dẫn đến mục tiêu để đi đến thành công;

(4) Khả năng tự phục hồi là khi đối mặt với khó khăn, nghịch cảnh thì bản thân luôn tự duy trì sự ổn định, tiếp tục khắc phục và thậm chí là vươn lên mạnh mẽ hơn để đạt được thành công (Luthans et al., 2007).

Như vậy có thể kết luận: Nguồn lực tâm lý (Psycap) được định nghĩa như một năng lực cốt lõi bậc cao, liên quan đến các đặc điểm về tâm lý tích cực (tự tin, lạc quan, hy vọng, khả năng tự phục hồi) hướng đến sự thành công, và không thể thay đổi được. (Avey, Wernsing, \& Luthans, 2008).

Các thành tố của nguồn lực tâm lý đều thể hiện mối quan hệ tích cực với cam kết trong tổ chức (Luthans, Norman, Avolio, \& Avey, 2008). Nghiên cứu thực nghiệm đã phát hiện ra rằng cam kết tổ chức có mối quan hệ tích cực với hi vọng, lạc quan, khả năng tự phục hồi, tự tin (Sinha, Talwar, \& Rajpal, 2002; Youssef \& Luthans, 2007).

Khi một cá nhân rất lạc quan về tương lai của họ trong tổ chức thì họ sẽ cam kết dùy trì công việc của họ (Larson \& Luthans, 2006). Trong một nghiên cứu của McColl-Kennedy và Anderson (2005) đã có bằng chứng cho mối liên kết phụ thuộc giữa sự lạc quan và cam kết tổ chức. Lạc quan, là một nguồn lực cá nhân, có thể được phát triển nhằm có kết quả tốt về thái độ như cam kết làm việc và cam kết tổ chức (Simons \& Buitendach, 2013). Bên cạnh đó, khi cá nhân có tự tin thì họ có thể kiểm soát cuộc sống của họ cũng như có thể đóng góp cho các tổ chức (Larson \& Luthans, 2006). Ngoài ra, khi cá nhân có niềm hy vọng thì họ sẽ cảm thấy có động lực 
để thành công dù bất kỳ nhiệm vụ nào mà họ phải thực hiện trong tổ chức (Luthans, 2002). Hy vọng là một trong những thành phần của nguồn lực tâm lý, là một trạng thái động lực bao gồm cả quyết tâm xác định những mục tiêu cao cả và tin tưởng vượt qua được những trở ngại để đạt những mục tiêu này. Nhân viên hy vọng nhiều hơn cũng có thể hài lòng hơn với công việc của họ và cam kết nhiều hơn với các tổ chức của họ (Çetin, 2011). Như vậy, từ những nghiên cứu trên cho thấy tồn tại mối quan hệ tích cực giữa nguồn lực tâm lý và cam kết trong tổ chức.

\section{H1: Nguồn lực tâm lý có tác động đến sự cam kết với tổ chức}

Zamahani, Ghorbani, và Rezaei (2011) đã kiểm tra các mối quan hệ giữa nguồn lực tâm lý và kết quả công việc trên mẫu của 200 nhân viên của một công ty viễn thông lớn tại Iran. Kết quả cho thấy một mối tương quan tích cực và có ý nghĩa thống kê giữa nguồn lực tâm lý và kết quả công việc của nhân viên công việc $(\mathrm{r}=.48, \mathrm{p}<.001)$. Bằng việc sử dụng mẫu là 422 người lao động tại Trung Quốc tại 3 nhà máy khác nhau, Luthans, Avolio, Walumbwa, và Li (2005) cũng đã tìm thấy mối tương quan tích cực giữa nguồn lực tâm lý và kết quả công việc của nhân viên $(\mathrm{r}=$ $.26, \mathrm{p}<.01$ ). Tương tự với 456 mẫu từ 2 nhà máy luyện đồng của Trung Quốc (một của tư nhân, một của nhà nước), phân tích cũng cho kết quả ủng hộ mối quan hệ giữa nguồn lực tâm lý và kết quả công việc (Luthans et al., 2008). Mối tương quan giữa hai biến là tích cực và có ý nghĩa thống kê $(r=.25, \mathrm{p}<.01)$.

Walumbwa, Luthans, Avey, và Oke (2011) cũng tìm ra được mối quan hệ tích cực và có ý nghĩa thống kê giữa 2 yếu tố này, nhưng trên mức độ nhóm thay vì trên mức độ cá nhân. Nhóm tác giả này đã sử dụng 146 nhóm làm việc bao gồm khoảng 526 nhân viên của một ngân hàng lớn, kết quả của họ đã cho thấy mối tương quan tích cực giữa nguồn lực tâm lý và kết quả công việc của nhóm theo đánh giá của các trưởng nhóm $(\mathrm{r}=.39, \mathrm{p}<.01)$.

\section{H2: Nguồn lực tâm lý có tác động đến Kết quả làm việc của nhân viên}

\subsection{Sụ̂ cam kết với tổ chức}

Các khái niệm về cam kết của tổ chức (Organizational Commitment - OC) đã được phát triển phổ biến trong các nghiên cứu mang tính khoa học xã hội (Chughtai \& Zafar, 2006). Mối quan tâm về yếu tố này được thể hiện ở cả mặt lý thuyết để giải thích các cấu trúc của sự cam kết và cả mặt thực nghiệm để xác định mức độ cam kết và kết quả của sự cam kết với tổ chức của nhân viên (Kanter, 1977; Mowday, Porter, \& Dubin, 1974; Porter, Steers, Mowday, \& Boulian, 1974; Salancik, 1977; Steers, 1977). Sự cam kết tổ chức giữa vai trò quan trọng trong nghiên cứu hành vi tích cực của nhân viên trong tổ chức (Griffin \& Bateman, 1986; Meyer, Stanley, Herscovitch, \& Topolnytsky, 2002; Meyer \& Herscovitch, 2001; Reichers 1985) và là yếu tố gắn kết giữa nhân viên và tổ chức, giúp tổ chức thành công trên thị trường (Fornes, Rocco, \& Wollard, 2008). Các nghiên cứu cho thấy Sự cam kết không những có tác động trực tiếp đến kết quả làm việc của nhân viên (Chen, Silverthorne, \& Hung, 2006), sự hài lòng trong công việc (Chughtai \& Zafar, 2006) mà còn ảnh hưởng tích cực đến hành vi công dân trong tổ chức (Zeinabadi \& Salehi, 2011).

Các khái niệm về cam kết tổ chức cũng như cách đo lường đã được đề xuất và thử nghiệm theo nhiều khía cạnh khác nhau (Meyer \& Allen, 1991; Mowday, Porter, \& Steers, 1982). Sự khác biệt của các khái niệm xuất phát từ nền tảng tâm lý được phản ánh trong sự cam kết hay điều kiện tiền đề dẫn đến sự phát triển của nó cũng như những hành vi được mong đợi từ kết quả của cam kết tạo ra (Meyer \& Allen, 1991). Sự cam kết theo quan điểm trao đổi xã hội (Eisenberger, Huntington, Hutchison, \& Sowa, 1986) chính là nhận thức của người lao động về cam kết của tổ chức đối với họ (nhận thức về sự hỗ trợ của tổ chức). Eisenberger và cộng sự (1986) cho rằng những nhân viên cảm nhận được mức độ hỗ trợ cao từ tổ chức thì sẽ thấy có nghĩa vụ phải đóng 
góp lại cho tổ chức. Điều đó nằm trong phạm trù thuộc về các cam kết tình cảm và có hành vi làm việc tích cực liên quan (Blau, 1964; Eisenberger et al.,1986).

Meyer và Allen (1987) cho rằng Sự cam kết như một trạng thái tâm lý phản ánh mối quan hệ của nhân viên với tổ chức, và được chia thành ba thành phần chính: gắn kết tình cảm (affective attachment), những chi phí cảm nhận được (perceived costs) và nghĩa vụ (obligation).

(1) Gắn kết tình cảm là cách tiếp cận phổ biến nhất đến cam kết tổ chức, được xem là những tình cảm, cảm xúc gắn bó mạnh mẽ với tổ chức mà cá nhân cảm nhận được và sự thích thú khi tham gia, muốn trở thành thành viên của tổ chức (Buchanan, 1974).

(2) Chi phí được cảm nhận là nhận thức của cá nhân về các 'chi phí' phải bỏ ra cho việc ngừng hoạt động (Becker, 1960; Rusbult \& Farrell, 1983). Hay có thể nói rằng 'cam kết nhận thức về việc tiếp tục' ở lại tổ chức xuất hiện khi thấy được "lợi nhuận sinh ra nếu tiếp tục tham gia và chi phí sinh ra nếu rời khỏi” (Kanter, 1968).

(3) Nghĩa vụ là niềm tin về trách nhiệm của một người trong tổ chức. Wiener (1982) quy định cam kết là "toàn bộ những áp lực của bản quy phạm buộc nhân viên phải hành động một cách đúng đắn với các mục tiêu và lợi ích của tổ chức”, và cho thấy rằng hành vi cá nhân biểu hiện chỉ là vì “họ tin rằng đó là ‘đúng' là đạo đức, là điều phải làm”.

Ba cách tiếp cận này đưa ra cái nhìn tốt nhất về các thành phần của cam kết về thái độ (Meyer \& Allen, 1990) và được đặt tên là cam kết 'tình cảm', cam kết 'tài chính' và cam kết 'quy phạm' (Meyer \& Allen, 1987). Những nhân viên với cam kết tình cảm mạnh mẽ ở lại tổ chức vì họ "muốn", cam kết tiếp tục mạnh mẽ vì họ "cần”, cam kết quy phạm mạnh mẽ vì họ cảm thấy họ nên làm như vậy. Nghiên cứu lựa chọn theo hướng của nghiên cứu Meyer và Allen (1990) và tập trung chủ yếu vào thành phần cam kết tình cảm, một yếu tố chi phối nhiều mặt trong cuộc sống của con người, được đánh giá là yếu tố mang tính đại diện cao cho Sự cam kết với tổ chức và được sử dụng trong nhiều nghiên cứu về Sự cam kết (Feather \& Rauter, 2004; Lee \& Bruvold, 2003).

Các nghiên cứu trước đây đã khẳng định rằng giữa cam kết tổ chức và kết quả công việc có mối quan hệ tích cực (Chi, Tsai, \& Chang, 2007; Meyer et al., 2002; Suliman \& Iles, 2000). Morris và Sherman (1981) đã chỉ rõ: cam kết tổ chức có tác dụng trong việc dự đoán kết quả công việc. Mặt khác, Meyer, Paunonen, Gellatly, Goffin, và Jackson (1989) đã phát hiện ra rằng cam kết tổ chức là một chỉ số phù hợp của kết quả làm việc của nhân viên. Ngoài ra, Mowday và cộng sự (1974), Baugh và Roberts (1994), Ward và Davis (1995) cũng đã kết luận rằng cam kết trong tổ chức có tác động dương đến kết quả công việc.

Brett, Cron, và Slocum (1995) đã chứng minh mối quan hệ này bằng cách sử dụng các biến kinh tế phụ thuộc trong công việc như một biến trung gian. Nghiên cứu đã chỉ ra có một mối quan hệ mạnh mẽ giữa cam kết tổ chức với kết quả công việc đối với người có yêu cầu về tài chính thấp hơn so với người có yêu cầu tài chính cao. Putti, Aryee, và Liang (1989) cũng nghiên cứu mối quan hệ giữa kết quả công việc với cam kết tình cảm và cam kết tài chính. Kết quả nghiên cứu cho thấy, thông qua nhận thức về tổ chức, các cá nhân hình thành cam kết về tình cảm. Từ đó, cá nhân có sự gắn kết về mặt tình cảm với tổ chức tạo ra kết quả công việc cao hơn so với những đồng nghiệp khác. Việc cá nhân nhận thức về tổ chức hay công việc theo hướng tích cực hay tiêu cực được quyết định bởi những yếu tố tâm lý tích cực của nhân viên (Luthans et al., 2007). Như vậy, một cá nhân có tâm lý tích cực sẽ nhận thức vấn đề theo hướng lạc quan, có được trạng thái vui vẻ trong quá trình làm việc và từ đó hình thành nên sự gắn kết tình cảm với nơi làm việc. Sự gắn kết tình cảm cao lại là nhân tố quyết định hiệu quả làm việc của nhân viên. Có thể thấy, tâm lý tích cực có thể ảnh hưởng đến kết quả làm việc của nhân viên thông qua sự cam kết với tổ chức. Điều này cũng được ủng hộ trong nghiên cứu của Xizhou và Xiaoyan (2013), kết quả nghiên cứu đã cho 
thấy mối quan hệ tổng hợp giữa nguồn lực tâm lý và sự cam kết đối với kết quả làm việc của nhân viên. Nguồn lực tâm lý giúp gia tăng sự cam kết của nhân viên và từ đó giúp làm gia tăng kết quả làm việc của họ.

H3: Sự cam kết với tổ chức có tác động đến Kết quả làm việc của nhân viên

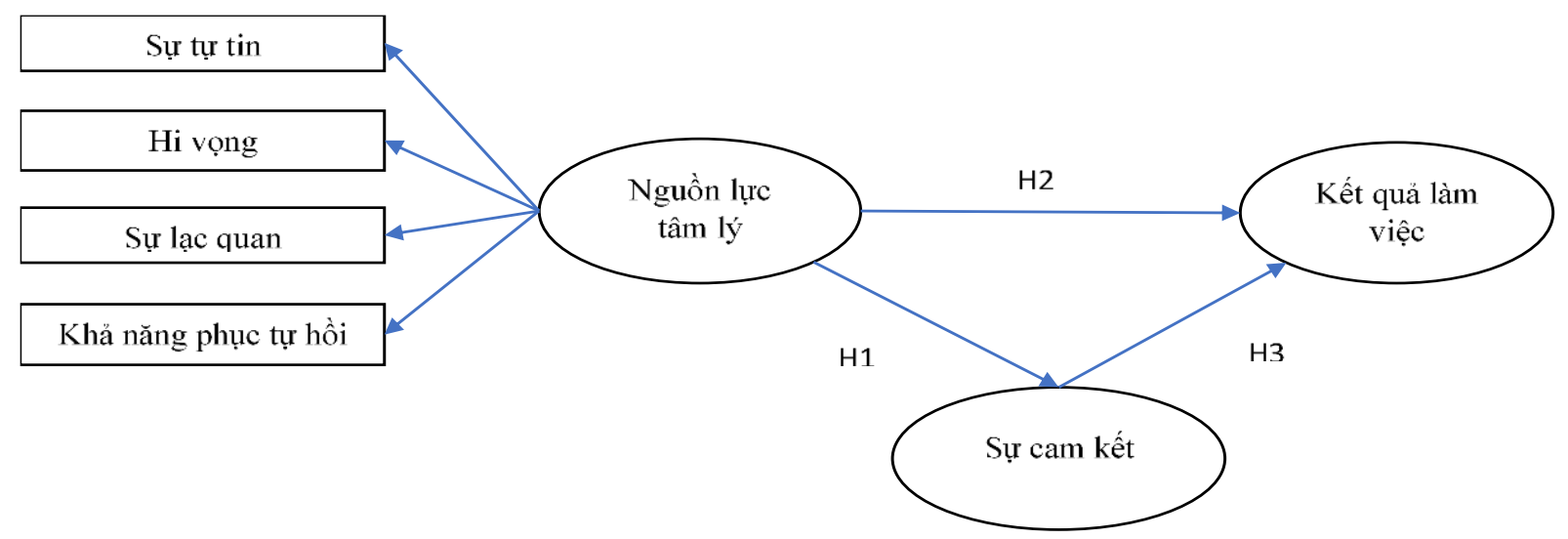

Hình 1. Mô hình nghiên cứu đề xuất

\section{Phương pháp nghiên cứu}

\subsection{Phương pháp nghiên cúu}

Nghiên cứu sử dụng phương pháp định lượng là chủ yếu kết hợp với phương pháp định tính trong nghiên cứu sơ bộ. Ban đầu, việc tổng hợp cơ sở lý thuyết, xây dựng mô hình và thiết lập thang đo được thực hiện. Sau đó, nghiên cứu định tính được triên khai thông qua phương pháp phỏng vấn chuyên gia và phỏng vấn nhóm tập trung nhằm hiệu chỉnh thang đo và làm rõ các biến quan sát sao cho phù hợp với hoàn cảnh và nhận thức của đối tượng được khảo sát. Phỏng vấn chuyên gia được thực hiện nhằm đảo bảo việc chuyển ngữ (từ tiếng Anh sang Việt) của thang đo được đảm bảo độ chính xác, không gây nhầm lẫn. Sau khi bảng câu hỏi nháp được hoàn thiện, phỏng vấn nhóm tập trung được thực hiện với mục tiêu kiểm định lại mức độ chính xác của bảng câu hỏi trong việc khai thác thông tin từ đối tượng khảo sát. Nhóm 10 thành viên được thành lập. Đối tượng tham gia nhóm là những nhân viên làm việc tại khu vực Thành phố Hồ Chí Minh. Các ý kiến của đối tượng phỏng vấn được ghi chép lại nhằm phục vụ cho việc hoàn thiện bảng câu hỏi khảo sát cuối cùng.

Phương pháp nghiên cứu định lượng được thực hiện trong nghiên cứu chính thức. Dữ liệu sau khi mã hóa được phân tích bằng phần mềm SPSS để kiểm tra hệ số tin cậy Cronbach's Alpha và phân tích khám phá nhân tố (EFA). Phần mềm Amos sẽ được sử dụng trong phân tích khẳng định nhân tố (CFA) và phân tích mô hình cấu trúc tuyến tính (SEM).

\subsection{Thang đo sü dụng trong nghiên cúu}

Thang đo nguồn lực tâm lý được sử dụng trong nghiên cứu là thang đo của Youssef và Luthans (2007) gồm 24 biến quan sát. Luthans, Youssef, và Avolio (2007) đã xây dựng thang đo Nguồn lực tâm lý bằng việc tổng hợp những thang đo trước đó về 4 yếu tố bao gồm: (a) Sự hy vọng (Snyder et al., 1996); (b) Sự tự hồi phục (Wagnild \& Young, 1993); (c) Sự lạc quan (Scheier \& Carver, 1985); và (d) Sự tự tin (Parker, 1998). Đây là thang đo Likert 6 điểm từ 1: "Hoàn toàn không đồng ý" đến 6: "Hoàn toàn đồng ý". Kết quả kiểm định trong các nghiên cứu trước đây cho thấy đây là thang đo có độ tin cậy cao và đã được sử dụng rộng rãi trong nghiên cứu liên quan đến nguồn lực tâm lý. 
Thang đo Cam kết tình cảm được trích từ thang đo cam kết trong tổ chức (Organizational Commitment) của Allen và Meyer (1990) gồm 24 biến quan sát, được dựa trên nền tảng thang đo OCQ của Mowday, Steers, và Porter (1979). Đây là thang đo Likert 7 điểm trong đó 1: "hoàn toàn không đồng ý" và 7: "hoàn tòan đồng ý". Tuy nhiên, thành phần cam kết tình cảm được sử dụng khá phổ biến trong việc đại diện cho khái niệm Sự cam kết (Feather \& Rauter, 2004; Lee \& Bruvold, 2003). Các nghiên cứu cũng cho thấy Cam kết tình cảm có tác động trực tiếp đến Kết quả công việc và là biến chịu sự tác động trực tiếp từ đặc điểm tâm lý tích cực của nhân viên. Do đó, để phục vụ cho nghiên cứu này tác giả chỉ sử dụng 8 biến quan sát thuộc thành phần Cam kết tình cảm.

Thang đo kết quả công việc với 4 biến quan sát dựa trên nghiên cứu của Stevens, Beyer, và Trice (1978) và Yousef (2000) với 5 mức độ đánh giá từ 1: "Rất thấp" đến 5: "Rất cao". Để người tham gia khảo sát có thể dễ hiểu và hiểu đúng ý nghĩa của từng biến quan sát, nhóm nghiên cứu đã có sự thay đổi về mặt kết cấu câu (từ câu nghi vấn sang câu khẳng định), cũng như thang đo là từ 1: "Hoàn toàn không đồng ý" đến 5: "Rất đồng ý".

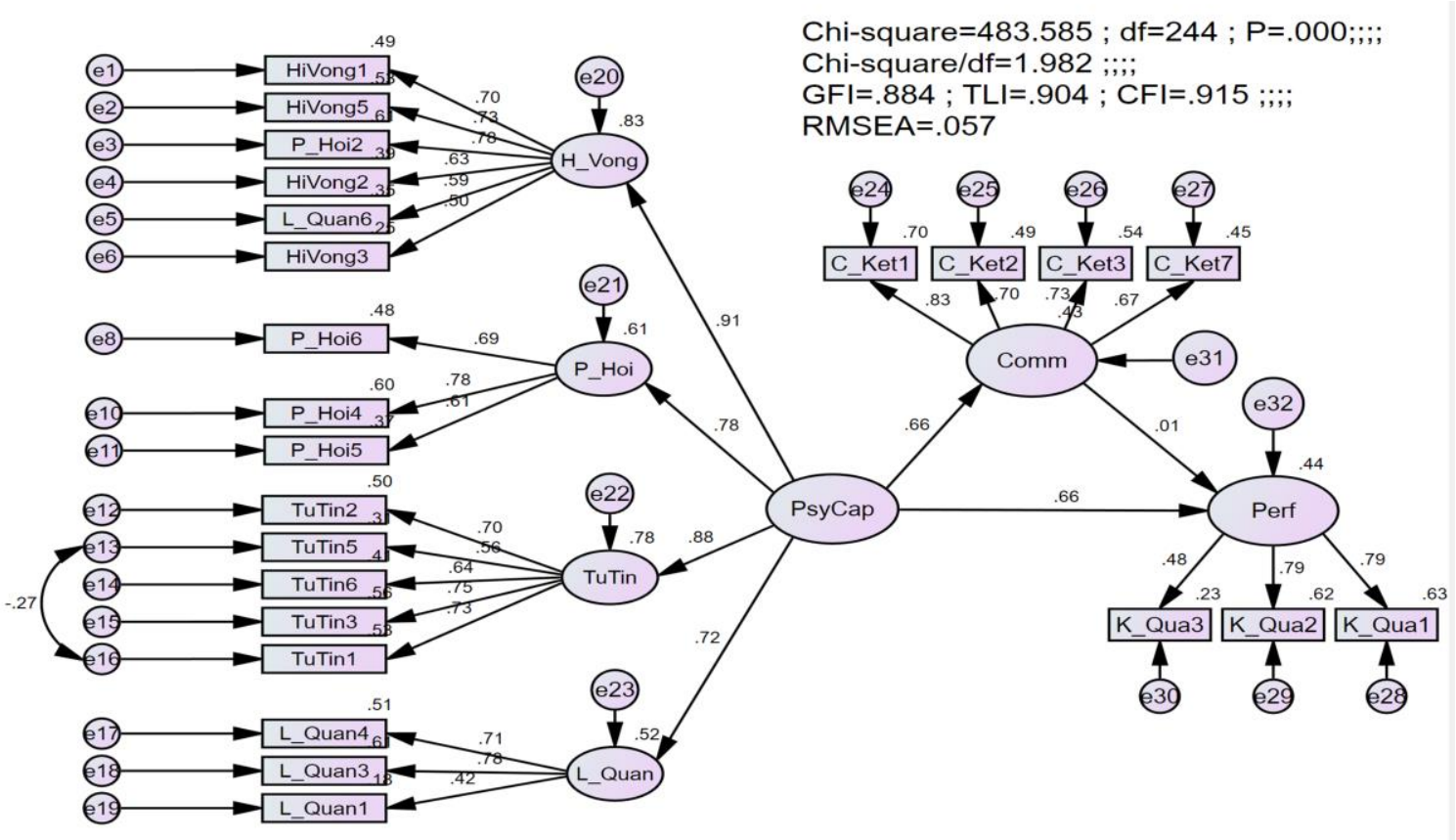

Hình 2. Mô hình SEM (đã chuẩn hóa)

\subsection{Mẫu nghiên cứu}

Trong trường hợp sử dụng phương pháp phân tích nhân tố (EFA) thì kích thước mẫu tối thiểu phải là 50 , tốt hơn là 100 và tỉ lệ số quan sát trên biến đo lường là $5: 1$ nghĩa là cứ mỗi biến đo lường cần tối thiểu là 5 quan sát (Hair, Black, Babin, Anderson, \& Tatham, 2006). Số biến được xác định trong nghiên cứu định lượng là 36, do đó số quan sát tương ứng là 180.

Khi tăng kích thước mẫu, độ tin cậy càng tăng, vì vậy tác giả quyết định kích thước mẫu là 300 để có một sự chính xác tương đối trong kết quả phân tích. Tuy nhiên để có được mẫu với số lượng là 300 , tác giả đã phát ra 350 bảng câu hỏi.

Đối tượng khảo sát là các nhân viên văn phòng làm việc tại các doanh nghiệp ở thành phố Hồ Chí Minh. Do điều kiện về thời gian và chi phí, mẫu được lựa chọn theo phương pháp thuận tiện, phi xác suất. Sau khi phát ra, số lượng phiếu thu về là 340 , trong đó có 36 phiếu không hợp lệ do bỏ sót câu trả lời, đánh một câu trả lời cho toàn bộ bảng khảo sát. Kết quả còn lại là 304 mẫu hợp lệ được sử dụng cho nghiên cứu. 


\section{Kết quả và đánh giá}

\subsection{Kết quả nghiên cúu}

Trong 304 phiếu khảo sát thu về thì nam chiếm 43,8\%, nữ chiếm 55,9\%; độ tuổi từ 18 - 22 chiếm 21,7\%, 23 - 30 tuổi chiếm 57,2\%, 31 - 40 tuổi chiếm 14,2\%, trên 40 tuổi chiếm 6,6\%; trình độ Trung học phổ thông chiếm 6,3\%, Đại học chiếm 76,6\%, Cao học chiếm 6,6\% và nhóm khác chiếm 10,2\%; nhân viên chiếm 72,1\%, quản lý chiếm 13,5\%, nhóm khác chiếm 14,1\%.

Hệ số Cronbach's Alpha được sử dụng để dánh giá độ tin cậy của thang đo. Các biến quan sát có hệ số tương quan biến tổng nhỏ hơn 0,3 không đạt yêu cầu về đo lường và sẽ bị loại khỏi thang đo khi đưa vào phân tích EFA. Phân tích EFA và $C F A$ được tiến hành để phân tích khám phá nhân tố, khẳng định giá trị hội tụ và phân biệt của thang đo. Mô hình đo lường và mô hình cấu trúc cũng được kiểm định thông qua phương pháp phân tích mô hình cấu trúc tuyến tính SEM.

Thành phần "Sự tự tin" có hệ số Cronbach's Alpha cao $(0,818)$ và các hệ số tương quan biến tổng đều lớn hơn 0,45 . Thành phần "sự hy vọng” cũng có hệ số Cronbach's Alpha cao $(0,789)$ và các hệ số tương quan biến tổng đều lớn hơn 0,39 . Các thang đo này có độ tin cậy cao và có ý nghĩa thống kê. Do đó các biến đều được giữ nguyên và đưa vào phân tích EFA.

Thành phần "khả năng tự phục hồi" có hệ số Cronbach's Alpha $(0,707)$, trong đó biến P_Hoil có hệ số tương quan biến tổng thấp $(0,093)$ nên tiến hành loại bỏ. Sau đó thu được kết quả với hệ số Cronbach's Alpha cao $(0,792)$ và các hệ số tương quan biến tổng đều lớn hơn 0,50 . Đồng thời thành phần "Sự lạc quan" cũng có hệ số Cronbach's Alpha rất thấp $(0,279)$, loại bỏ lần lượt 2 biến L_Quan2 vì có hệ số tương quan biến tổng: -0,233, L_Quan5 có hệ số tương quan biến tổng: 0,005 . Sau khi loại bỏ hai biến thì hệ số Cronbach's Alpha là 0,696 và các hệ số tương quan biến tổng đều lớn hơn 0,35 . Các thang đo có độ tin cậy khá cao và có ý nghĩa thống kê. Do đó các biến đạt còn lại được đưa vào phân tích EFA. Vậy có 3 biến bị loại trước khi đưa vào phân tích EFA là P_Hoi1, L_Quan2, L_Quan5.

Thang đo nguồn lực tâm lý còn lại 21 biến được đưa vào phân tích EFA. Kết quả cuối cùng

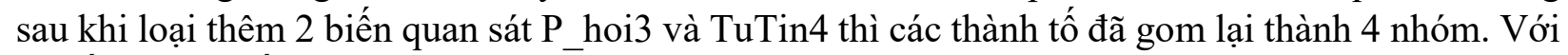
hệ số KMO's rất cao là 0,905 và có mức ý nghĩa Sig là 0,000 (nhỏ hơn 0,05 ), như vậy phân tích EFA được sử dụng ở đây là thích hợp.

Sử dụng phương pháp trích nhân tố Principal Components với phép quay Varimax, kết quả cho thấy tất cả các biến đều có hệ số tải nhân tố đạt yêu cầu (lớn hơn 0,5 ). Tại mức giá trị Eigenvalue $=1,054$ (lớn hơn 1 ) và tổng phương sai trích đạt được là 57,809\% (lớn hơn $50 \%$ ), từ 19 biến chia thành 4 nhân tố. Vì để đảm bảo giá trị nội dung các biến quan sát, nên giữ nguyên tên các thành phần của nguồn lực tâm lý: Hy vọng, khả năng tự phục hồi, tự tin, lạc quan.

\section{Bảng 1}

Kết quả kiểm định mối quan hệ nhân quả giữa các khái niệm (chưa chuẩn hóa)

\begin{tabular}{cccccccc} 
Giả thuyết & & & & Uoóc lưọng & S.E. & C.R. & P \\
\hline H1 & COMM & $<--$ & PSYCAP & 1.059 & 0.124 & 8.571 & $* * *$ \\
\hline H2 & JOBP & $<--$ & PSYCAP & 0.594 & 0.092 & 6.423 & $* * *$ \\
\hline H3 & JOBP & $<---$ & COMM & 0.008 & 0.05 & 0.156 & 0.876
\end{tabular}


Thang đo cam kết tình cảm: do hệ số Cronbach's Alpha ban đầu thấp $(0,588)$ nên sau khi loại bỏ lần lượt một số biến quan sát: C_Ket4, C_Ket5, C_Ket6, C_Ket8 (loại biến C_Ket4 trước vì có hệ số tương quan biến tổng thấp nhất: $0,06 \overline{2}$ ) thì hệ số Cronbach's Alpha là $0,8 \overline{2} 2$ và các hệ số tương quan biến tổng đều lớn hơn 0,59 . Thang đo này có độ tin cậy cao và có ý nghĩa thống kê. Do đó các biến còn lại được đưa vào phân tích EFA.

Thang đo kết quả công việc có hệ số Cronbach's Alpha khi chạy lần đầu là rất thấp 0,477. Nên sau khi phân tích loại bỏ biến quan sát K_Qua4 (hệ số tương quan biến tổng là 0,002 ), hệ số Cronbach's Alpha là 0,693 và hệ số tương quan biến tổng các biến còn lại đều lớn hơn 0,39 . Thang đo có độ tin cậy khá cao và có ý nghĩa thống kê.

\section{Kiểm định CFA và mô hình SEM}

Kiểm định CFA ban đầu có 292 bậc tự do với Chi bình phương là 673,009, $\mathrm{P}=0,000$. Mô hình phù hợp với dữ liệu thị trường qua các chỉ số $\mathrm{CMIN} / \mathrm{df}=2,305<3 ; \mathrm{GFI}=0,855$; TLI=0,868; $\mathrm{CFI}=0,881$ và $\mathrm{RMSEA}=0,066<0,08$. Vì các chỉ số GFI, TLI, CFI đều bé hơn 0,9 nên mô hình này chưa phù hợp với dữ liệu thu thập. Do đó tiến hành hiệu chỉnh mô hình và thu được kết quả mô hình có độ tin cậy khá cao.

Sau khi tiến hành hiệu chỉnh, mô hình có 244 bậc tự do với Chi bình phương là 483,585, $\mathrm{P}=0,000$. Mô hình phù hợp với dữ liệu thị trường qua các chỉ số $\mathrm{CMIN} / \mathrm{df}=1,982<3 ; \mathrm{GFI}=0,884$; $\mathrm{TLI}=0,904 ; \mathrm{CFI}=0,915$ đều gần như lớn hơn hoặc gần bằng 0,9 và $\mathrm{RMSEA}=0,057<0,08$.

Qua phân tích cấu trúc tuyến tính SEM, mô hình có 244 bậc tự do với các giá trị thống kê như sau: Chi bình phương là 483,585, $\mathrm{P}=0,000, \mathrm{CMIN} / \mathrm{df}=1,982, \mathrm{GFI}=0,884, \mathrm{TLI}=0,904, \mathrm{CFI}=0,915$ và $\mathrm{RMSEA}=0,057$. Kết quả này cho thấy mô hình điều chỉnh phù hợp với dữ liệu thống kê.

Kết quả ước lượng đã chuẩn hóa các tham số được thể hiện tại Bảng 1 cho thấy nguồn lực tâm lý có tác động dương đến cam kết tình cảm (PSYCAP - COMM). Hệ số hồi quy chuẩn hóa của mối quan hệ $\mathrm{COMM} \leftarrow \mathrm{PSYCAP}$ là 0,657 , với mức ý nghĩa thống kê $\mathrm{P}<0,05$. Do đó, giả thuyết $\mathrm{H} 1$ được chấp nhận. Điều này có nghĩa là cứ một đơn vị tăng/giảm Nguồn lực tâm lý sẽ làm tăng/giảm 0,657 đơn vị Cam kết tình cảm. Ngoài ra, dựa vào hệ số bình phương tương quan bội qua phân tích SEM cho thấy nguồn lực tâm lý giải thích được 43,2\% biến thiên dữ liệu của cam kết tình cảm.

Giữa nguồn lực tâm lý và kết quả công việc (PSYCAP - JOBP) có mối quan hệ cùng chiều. Hệ số hồi quy chuẩn hóa của mối quan hệ JOBP $\leftarrow$ PSYCAP là 0,648 , với mức ý nghĩa thống kê $\mathrm{P}<0,05$. Như vậy, giả thuyết $\mathrm{H} 2$ được chấp nhận. Khi Nguồn lực tâm lý tăng/giảm một đơn vị sẽ làm tăng/giảm 0,648 đơn vị kết quả công việc. Hệ số bình phương tương quan bội qua phân tích SEM cho thấy nguồn lực tâm lý giải thích được $43,7 \%$ biến thiên dữ liệu của kết quả làm việc.

Tuy nhiên trong mối quan hệ giữa cả ba thành tố nguồn lực tâm lý, cam kết tình cảm và kết quả công việc thì mối quan hệ giữa COMM - JOBP là không có ý nghĩa thống kê. Hệ số hồi quy chuẩn hóa của mối quan hệ JOBP $\leftarrow$ COMM là 0,019 , với mức ý nghĩa thống kê $\mathrm{P}=0,876$ (> $0,05)$. Do đó, giả thuyết H3 không được chấp nhận.

\section{So sánh sụ khác biệt theo giới tính}

Mô hình bất biến và mô hình khả biến được sử dụng để so sánh. Kết quả phân tích SEM trong bảng 2 cho thấy sự khác biệt giữa hai mô hình không có ý nghĩa thống kê ( $\mathrm{p}=0,782>0,05)$. Như vậy, ta chọn mô hình bất biến. Hay nói cách khác, giới tính không làm ảnh hưởng đến mối quan hệ giữa nguồn lực tâm lý, sự cam kết và kết quả làm việc của nhân viên. 


\section{Bảng 2}

Sự khác biệt các chỉ tiêu tương thích của mô hình bất biến và khả biến theo giới tính

\begin{tabular}{|c|c|c|c|c|c|c|}
\hline Mô hình & Chi-Square & df & $\mathbf{p}$ & RFI & IFI & TLI \\
\hline Khả biến & 877,359 & 488 & 0,000 & 0,719 & 0,872 & 0,852 \\
\hline Bất biến & 878,439 & 491 & 0,000 & 0,721 & 0,873 & 0,854 \\
\hline Giá trị khác biệt & 1,08 & 3 & 0,000 & 0,002 & 0,001 & 0,002 \\
\hline
\end{tabular}

Nguồn: Kết quả phân tích dữ liệu của nhóm nghiên cứu

\section{2. Đánh giá}

Kết quả nghiên cứu cho thấy thang đo về nguồn lực tâm lý, sự cam kết về tình cảm với tổ chức đã có sự biến đổi khi kiểm định tại Việt Nam. Cụ thể, khái niệm sự cam kết tình cảm được đo lường bằng 8 biến quan sát đã được thay đổi còn 4 biến quan sát để đảm bảo độ tin cậy của thang đo. Khái niệm nguồn lực tâm lý theo thang đo gốc của Luthans và cộng sự (2007) được đo lường bằng 24 biến quan sát sau khi phân tích CFA đã được rút gọn xuống 17 biến quan sát với độ tin cậy và giá trị đo lường cao. Có sự dịch chuyển của một số khái niệm thuộc nhân tố phục hồi, lạc quan sang nhân tố hi Vọng. Bốn thành tố của nguồn lực tâm lý vẫn được giữ nguyên.

Mô hình SEM cho thấy nguồn lực tâm lý có tác động tích cực đến sự cam kết của nhân viên. Kết quả nghiên cứu phù hợp với lý thuyết và tương đồng với khám phá của Simons và Buitendach (2013), Kim, Seo, Kim, và Min (2015). Như vậy, nhân viên với nguồn lực tâm lý tích cực sẽ hình thành nên những định hướng suy nghĩ lạc quan trong công việc, hình thành niềm tin và gắng kết tình cảm với tổ chức. Mặt khác, phân tích mô hình cấu trúc tuyến tính đã cho thấy tồn tại mối quan hệ thuận chiều giữa nguồn lực tâm lý và kết quả công việc của nhân viên. Kết quả giúp khẳng định mối quan hệ đã được chứng minh trong các nghiên cứu về nguồn lực tâm lý và kết quả công việc trước đây (Luthans et al., 2005; Walumbwa et al., 2011; Zamahani et al., 2011). Ngoài ra, khi xét riêng mối quan hệ giữa sự cam kết và kết quả làm việc thì tồn tại sự tác động thuận chiều mang ý nghĩa thông kê. Tuy nhiên, giả thuyết về sự tác động của yếu tố sự cam kết đến kết quả làm việc trong mô hình lại bị bác bỏ khi xét đồng thời vai trò của nguồn lực tâm lý. Nói cách khác, sự cam kết tình cảm không đóng vai trò trung gian trong mối quan hệ giữa nguồn lực tâm lý và kết quả công việc của nhân viên. Do đó, cần có một nghiên cứu chuyên sâu tiếp theo để kiểm định lại và làm rõ sự khác biệt này.

Nghiên cứu chỉ thực hiện với mẫu 304 nhân viên tại khu vực Thành phố Hồ Chí Minh nên tính đại diện chưa cao. Khi nghiên cứu với số lượng đối tượng khảo sát lớn hơn hay thay đổi thời điểm, địa điểm nghiên cứu có thể sẽ ảnh hưởng đến mối quan hệ và mức độ tác động giữa các yếu tố.

Bên cạnh đó, thang đo được chuyển ngữ nên có thể chưa đảm bảo được sự chính xác hoàn toàn về nội dung. Thang đo gốc được xây dựng trong bối cảnh nền văn hóa nước ngoài nên có những biến quan sát chưa phù hợp để đo lường trong bối cảnh tại Việt Nam.

Các nghiên cứu tiếp theo có thể khảo sát thêm sự tác động đồng của nguồn lực tâm lý và các biến: sự hài lòng trong công việc, động lực nội sinh, động lực ngoại sinh, công bằng trong tổ chức, sự kháng cự với thay đổi... đến kết quả làm việc của nhân viên. Đặc biệt, vai trò trung gian của sự cam kết cần được kiểm định lại với vai trò là biến bậc 2 . Đồng thời phát triển mẫu với số lượng lớn để đem lại độ tin cậy và tính đại diện cao hơn. Ngoài ra, các nghiên cứu lập lại có thể được thực hiện ở những vùng miền, quốc gia khác nhau để kiểm định lại mối quan hệ giữa các khái niệm, củng cố thêm khám phá về mối quan hệ giữa các khái niệm trong mô hình nghiên cứu. 


\section{Kết luận}

Như vậy, với mức ý nghĩa 5\%, khái niệm nguồn lực tâm lý có tác động trực tiếp đến Sự cam kết và Kết quả làm việc của nhân viên trong tổ chức. Trong đó, mức tác động của nguồn lực tâm lý đến sự cam kết và kết quả công việc gần như bằng nhau $(\beta=0,66)$. Vai trò trung gian của sự cam kết tình cảm trong mối quan hệ giữa nguồn lực tâm lý và kết quả công việc không được ủng hộ.

Để nâng cao kết quả làm việc của nhân viên, các nhà quản trị cần tập trung nâng cao nguồn lực tâm lý của nhân viên. Cụ thể, gia tăng niềm hy vọng cho nhân viên thông qua việc chia sẻ cụ thể, rõ ràng mục tiêu của tổ chức đến từng nhân viên, để họ thấu hiểu và gắng kết giữa mục tiêu của tổ chức với mục tiêu cá nhân. Đồng thời quy định cụ thể những phần thưởng cho nhân viên khi hoàn thành tốt nhiệm vụ để nhân viên luôn hy vọng về phía trước mà không ngừng nỗ lực.

Trong môi trường cạnh tranh đầy khốc liệt như hiện nay, dù là trong loại hình doanh nghiệp nào thì người nhân viên phải thường xuyên đối mặt với khó khăn, thách thức. Điều này đòi hỏi những nhân viên phải có lòng tin vào bản thân mới có thể đương đầu và vượt qua những trở ngại ấy. Do đó, nhà quản trị cần phải thường xuyên tổ chức các chương trình giao lưu chia sẻ kinh nghiệm giữa các nhân viên cũng như cấp trên nhằm nâng cao năng lực, tạo sự kết nối giữa các thành viên với nhau trong công việc. Bằng cách đào tạo cho nhân viên cả về chuyên môn nghiệp vụ lẫn kỹ năng, nhà quản trị có thể giúp phát triển năng lực nhân viên, giúp họ tự tin đảm nhận vai trò của mình trong tồ chức.

Sự lạc quan cũng gần giống như hy vọng, lạc quan chính là luôn nhìn vào những mặt tốt, mặt tích cực của một sự việc. Điều này đòi hỏi nhân viên phải có một môi trường làm việc thoải mái, vui vẻ, bởi khi tâm trạng tốt thì con người sẽ kéo theo những các nhìn nhận vấn đề một cách tích cực. Chính bản thân nhưng người đứng đầu tổ chức/doanh nghiệp phải thể hiện sự lạc quan trong công việc, luôn có những nhìn nhận đúng đắn, tích cực để dần dần hình thành một nếp văn hóa cho tổ chức ấy.

\section{Tài liệu tham khảo}

Allen, N. J., \& Meyer, J. P. (1990). The measurement and antecedents of affective, continuance and normative commitment to the organization. Journal of Occupational Psychology, 63(1), 1-18.

Avey, J. B., Reichard, R. J., Luthans, F., \& Mhatre, K. H. (2011). Meta-analysis of the impact of positive psychological capital on employee attitudes, behaviors, and performance. Human Resource Development Quarterly, 22(2), 127-152.

Avey, J. B., Wernsing, T. S., \& Luthans, F. (2008). Can positive employees help positive organizational change? Impact of psychological capital and emotions on relevant attitudes and behaviors. The Journal of Applied Behavioral Science, 44(1), 48-70.

Baugh, S. G., \& Roberts, R. M. (1994). Professional and organizational commitment among engineers: Conflicting or complementing? IEEE Transactions On Engineering Management, 41(2), 108-114.

Becker, H. S. (1960). Notes on the concept of commitment. American Journal of Sociology, 66(1), $32-40$.

Blau, P. M. (1964). Justice in social exchange. Sociological Inquiry, 34(2), 193-206. 
Brett, J. F., Cron, W. L., \& Slocum, J. W., Jr. (1995). Economic dependency on work: A moderator of the relationship between organizational commitment and performance. Academy of Management Journal, 38(1), 261-271.

Buchanan, B. (1974). Building organizational commitment: The socialization of managers in work organizations. Administrative Science Quarterly, 19(4), 533-546.

Çetin, F. (2011). The effects of the organizational psychological capital on the attitudes of commitment and satisfaction: A public sample in Turkey. European Journal of Social Sciences, 21(3), 373-380.

Chen, J. C., Silverthorne, C., \& Hung, J. Y. (2006). Organization communication, job stress, organizational commitment, and job performance of accounting professionals in Taiwan and America. Leadership \& Organization Development Journal, 27(4), 242-249.

Chi, H. K., Tsai, H. P., \& Chang, P. F. (2007). Investigating the relationship among leadership styles, emotional intelligence and organization commitment on job performance: A study of salespeople in Thailand. The Journal of Human Resource and Adult Learning, 3(2), 199-212.

Chughtai, A. A., \& Zafar, S. (2006). Antecedents and consequences of organizational commitment among Pakistani university teachers. Applied HRM Research, 11(1), 39-64.

Eisenberger, R., Huntington, R., Hutchison, S., \& Sowa, D. (1986). Perceived organizational support. Journal of Applied Psychology, 71(3), 500-507.

Etebarian, A., Tavakoli, S., \& Abzari, M. (2012). The relationship between psychological capital and organizational commitment. African Journal of Business Management, 6(14), 5057-5060.

Feather, N. T., \& Rauter, K. A. (2004). Organizational citizenship behaviours in relation to job status, job insecurity, organizational commitment and identification, job satisfaction and work values. Journal of Occupational and Organizational Psychology, 77(1), 81-94.

Fornes, S. L., Rocco, T. S., \& Wollard, K. K. (2008). Workplace commitment: A conceptual model developed from integrative review of the research. Human Resource Development Review, 7(3), 339-357.

Griffin, R. W., \& Bateman, T. S. (1986). Job satisfaction and organizational commitment. In C. L. Cooper \& I. Robertson (Eds.), International review of industrial and oprganizational psychology (pp. 157-188). Chichester, IL: John Wiley \& Sons.

Hafiz, A. Z. (2017). Relationship between organizational commitment and employee's performance evidence from banking sector of Lahore. Arabian Journal of Business and Management Review, 7(2), 1-7.

Hair, J. F., Black, W. C., Babin, B. J., Anderson, R. E., \& Tatham, R. L. (2006). Multivariate data analysis. Upper Saddle River, NJ: Pearson University Press.

Kanter, R. M. (1968). Commitment and social organization: A study of commitment mechanisms in utopian communities. American Sociological Review, 33(4), 499-517.

Kanter, R. M. (1977). Some effects of proportions on group life. In The gender gap in psychotherapy (pp. 53-78). Boston, MA: Springer.

Khan, A. H., Nawaz, M. M., Aleem, M., \& Hamed, W. (2012). Impact of job satisfaction on employee performance: An empirical study of autonomous medical institutions of Pakistan. African Journal of Business Management, 6(7), 2697-2705. 
Kim, I. S., Seo, R. B., Kim, B. N., \& Min, A. R. (2015). The effects of positive psychological capital, organizational commitment, customer orientation in clinical nurses. Journal of Korean Academy of Nursing Administration, 21(1), 10-19.

Larson, M., \& Luthans, F. (2006). Potential added value of psychological capital in predicting work attitudes. Journal of Leadership \& Organizational Studies, 13(2), 75-92.

Lee, C. H., \& Bruvold, N. T. (2003). Creating value for employees: investment in employee development. The International Journal of Human Resource Management, 14(6), 981-1000.

Luthans, F. (2002). The need for and meaning of positive organizational behavior. Journal of Organizational Behavior: The International Journal of Industrial, Occupational and Organizational Psychology and Behavior, 23(6), 695-706.

Luthans, F., Avolio, B. J., Avey, J. B., \& Norman, S. M. (2007). Positive psychological capital: Measurement and relationship with performance and satisfaction. Personnel Psychology, 60(3), 541-572.

Luthans, F., Avolio, B. J., Walumbwa, F. O., \& Li, W. (2005). The psychological capital of Chinese workers: Exploring the relationship with performance. Management and Organization Review, 1(2), 249-271.

Luthans, F., Luthans, K. W., \& Luthans, B. C. (2004). Positive psychological capital: Beyond human and social capital. Management Department Faculty Publications, 47(1), 45-50.

Luthans, F., Norman, S. M., Avolio, B. J., \& Avey, J. B. (2008). The mediating role of psychological capital in the supportive organizational climate-employee performance relationship. Journal of Organizational Behavior: The International Journal of Industrial, Occupational and Organizational Psychology and Behavior, 29(2), 219-238.

Luthans, F., Youssef, C. M., \& Avolio, B. J. (2007). Psychological capital: Developing the human competitive edge. Oxford, UK: Oxford University Press.

Mayer, R. C., \& Schoorman, F. D. (1992). Predicting participation and production outcomes through a two-dimensional model of organizational commitment. Academy of Management Journal, 35(3), 671-684.

McColl-Kennedy, J. R., \& Anderson, R. D. (2005). Subordinate-manager gender combination and perceived leadership style influence on emotions, self-esteem and organizational commitment. Journal of Business Research, 58(2), 115-125.

Meyer, J. P., \& Allen, N. J. (1987). A longitudinal analysis of the early development and consequences of organizational commitment. Canadian Journal of Behavioural Science/Revue canadienne des sciences du comportement, 19(2), 199-215.

Meyer, J. P., \& Allen, N. J. (1991). A three-component conceptualization of organizational commitment. Human Resource Management Review, 1(1), 61-89.

Meyer, J. P., \& Herscovitch, L. (2001). Commitment in the workplace: Toward a general model. Human Resource Management Review, 11(3), 299-326.

Meyer, J. P., Paunonen, S. V., Gellatly, I. R., Goffin, R. D., \& Jackson, D. N. (1989). Organizational commitment and job performance: It's the nature of the commitment that counts. Journal of Applied Psychology, 74(1), 152-156.

Meyer, J. P., Stanley, D. J., Herscovitch, L., \& Topolnytsky, L. (2002). Affective, continuance, and normative commitment to the organization: A meta-analysis of antecedents, correlates, and consequences. Journal of Vocational Behavior, 61(1), 20-52. 
Morris, J. H., \& Sherman, J. D. (1981). Generalizability of an organizational commitment model. Academy of Management Journal, 24(3), 512-526.

Mowday, R. T., Porter, L. W., \& Dubin, R. (1974). Unit performance, situational factors, and employee attitudes in spatially separated work units. Organizational Behavior and Human Performance, 12(2), 231-248.

Mowday, R. T., Porter, L. W., \& Steers, R. (1982). Organizational linkages: The psychology of commitment, absenteeism, and turnover. San Diego, CA: Academic Press.

Mowday, R. T., Steers, R. M., \& Porter, L. W. (1979). The measurement of organizational commitment. Journal of Vocational Behavior, 14(2), 224-247.

Parker, S. K. (1998). Enhancing role breadth self-efficacy: The roles of job enrichment and other organizational interventions. Journal of Applied Psychology, 83(6), 835-852.

Peterson, S. J., Luthans, F., Avolio, B. J., Walumbwa, F. O., \& Zhang, Z. (2011). Psychological capital and employee performance: A latent growth modeling approach. Personnel Psychology, 64(2), 427-450.

Porter, L. W., \& Lawler, E. E. (1968). Managerial attitudes and performance. Homewood, IL: Irwin-Dorsey.

Porter, L. W., Steers, R. M., Mowday, R. T., \& Boulian, P. V. (1974). Organizational commitment, job satisfaction, and turnover among psychiatric technicians. Journal of Applied Psychology, 59(5), 603-609.

Pradeep, D. D., \& Prabhu, N. R. V. (2011). The relationship between effective leadership and employee performance. Journal of Advancements in Information Technology, 20(1), 198-207.

Putti, J. M., Aryee, S., \& Liang, T. K. (1989). Work values and organizational commitment: A study in the Asian context. Human Relations, 42(3), 275-288.

Reichers, A. E. (1985). A review and reconceptualization of organizational commitment. Academy of Management Review, 10(3), 465-476.

Rusbult, C. E., \& Farrell, D. (1983). A longitudinal test of the investment model: The impact on job satisfaction, job commitment, and turnover of variations in rewards, costs, alternatives, and investments. Journal of Applied Psychology, 68(3), 429-438.

Salancik, G. R. (1977). Commitment and the control of organizational behavior and belief. In B. M. Staw \& G. R. Salancik (Eds.), New directions in organizational behavior (pp. 1-54). Chicago, IL: St. Clair.

Scheier, M. F., \& Carver, C. S. (1985). Optimism, coping, and health: Assessment and implications of generalized outcome expectancies. Health Psychology, 4(3), 219-247.

Simons, J. C., \& Buitendach, J. H. (2013). Psychological capital, work engagement and organisational commitment amongst call centre employees in South Africa. SA Journal of Industrial Psychology, 39(2), 1-12.

Sinha, S. P., Talwar, T., \& Rajpal, R. (2002). Correlational study of organizational commitment, selfefficacy and psychological barriers to technological change. Psychologia, 45(3), 176-183.

Snyder, C. R., Sympson, S. C., Ybasco, F. C., Borders, T. F., Babyak, M. A., \& Higgins, R. L. (1996). Development and validation of the state hope scale. Journal of Personality and Social Psychology, 70(2), 321-335. 
Steers, R. M. (1977). Antecedents and outcomes of organizational commitment. Administrative Science Quarterly, 22(1), 46-56.

Stevens, J. M., Beyer, J. M., \& Trice, H. M. (1978). Assessing personal, role, and organizational predictors of managerial commitment. Academy of Management Journal, 21(3), 380-396.

Suliman, A., \& Al Harethi, B. (2013). Perceived work climate and employee performance in public security organizations in the UAE. Transforming Government: People, Process and Policy, $7(3), 410-424$.

Suliman, A., \& Iles, P. (2000). Is continuance commitment beneficial to organizations? Commitment-performance relationship: A new look. Journal of Managerial Psychology, 15(5), 407-422.

Wagnild, G. M., \& Young, H. (1993). Development and psychometric. Journal of Nursing Measurement, 1(2), 165-178.

Wall, T. D., Michie, J., Patterson, M., Wood, S. J., Sheehan, M., Clegg, C. W., \& West, M. (2004). On the validity of subjective measures of company performance. Personnel Psychology, 57(1), 95-118.

Walumbwa, F. O., Luthans, F., Avey, J. B., \& Oke, A. (2011). Authentically leading groups: The mediating role of collective psychological capital and trust. Journal of Organizational Behavior, 32(1), 4-24.

Ward, E. A., \& Davis, E. (1995). The effect of benefit satisfaction on organizational commitment. Compensation and Benefits Management, 11(3), 35-40.

Wiener, Y. (1982). Commitment in organizations: A normative view. Academy of Management Review, 7(3), 418-428.

Xizhou, T. I. A. N., \& Xiaoyan, Z. U. O. (2013). The synergic effects of human, social and psychological capital on hotel employee performance and organizational commitment. Tourism Tribune/Lvyou Xuekan, 28(11), 118-124.

Yousef, D. A. (2000). Organizational commitment: A mediator of the relationships of leadership behavior with job satisfaction and performance in a non-Western country. Journal of Managerial Psychology, 15(1), 6-24.

Youssef, C. M., \& Luthans, F. (2007). Positive organizational behavior in the workplace: The impact of hope, optimism, and resilience. Journal of Management, 33(5), 774-800.

Zamahani, M., Ghorbani, V., \& Rezaei, F. (2011). Impact of authentic leadership and psychological capital on followers' trust and performance. Australian Journal of Basic and Applied Sciences, 5(12), 658-667.

Zeinabadi, H., \& Salehi, K. (2011). Role of procedural justice, trust, job satisfaction, and organizational commitment in Organizational Citizenship Behavior (OCB) of teachers: Proposing a modified social exchange model. Procedia-Social and Behavioral Sciences, 29(2011), 1472-1481. 